\title{
Development of Anxiety-Like Behavior via Hippocampal IGF-2 Signaling in the Offspring of Parental Morphine Exposure: Effect of Enriched Environment
}

\author{
Chang-Qi Li', Yan-Wei Luo', Fang-Fang Bi' ${ }^{2}$ Tao-Tao Cui', Ling Song', Wen-Yu Cao', Jian-Yi Zhang', \\ Fang $\mathrm{Li}^{\prime}$, Jun-Mei $\mathrm{Xu}^{3}$, Wei $\mathrm{Hao}^{4}$, Xiao-Wei Xing ${ }^{5}$, Fiona $\mathrm{H}$ Zhou ${ }^{6}$, Xin-Fu Zhou ${ }^{6}$ and Ru-Ping Dai*,3 \\ 'Department of Anatomy and Neurobiology, Xiangya School of Medicine, Central South University, Changsha, Hunan Province, China; \\ ${ }^{2}$ Department of Neurology, Xiangya Hospital, Central South University, Changsha, Hunan Province, China; ${ }^{3}$ Department of Anesthesiology, The \\ Second Xiangya Hospital of Central South University, Changsha, Hunan Province, China; ${ }^{4}$ Institute of Mental Health, The Second Xiangya \\ Hospital of Central South University, Changsha, Hunan Province, China; ${ }^{5}$ Center for Medical Experiments, Third Xiang-Ya Hospital of Central \\ South University, Changsha, Hunan Province, China; ${ }^{6}$ School of Pharmacy and Medical Sciences, Division of Health Sciences, University of South \\ Australia, Adelaide, SA, Australia
}

\begin{abstract}
Opioid addiction is a major social, economic, and medical problem worldwide. Long-term adverse consequences of chronic opiate exposure not only involve the individuals themselves but also their offspring. Adolescent maternal morphine exposure results in behavior and morphologic changes in the brain of their adult offspring. However, few studies investigate the effect of adult opiate exposure on their offspring. Furthermore, the underlying molecular signals regulating the intergenerational effects of morphine exposure are still elusive. We report here that morphine exposure of adult male and female rats resulted in anxiety-like behavior and dendritic retraction in the dentate gyrus (DG) region of the hippocampus in their adult offspring. The behavior and morphologic changes were concomitant with the downregulation of insulin-like growth factor (IGF)-2 signaling in the granular zone of DG. Overexpression of hippocampal IGF-2 by bilateral intra-DG injection of lentivirus encoding the IGF-2 gene prevented anxiety-like behaviors in the offspring. Furthermore, exposure to an enriched environment during adolescence corrected the reduction of hippocampal IGF-2 expression, normalized anxietylike behavior and reversed dendritic retraction in the adult offspring. Thus, parental morphine exposure can lead to the downregulation of hippocampal IGF-2, which contributed to the anxiety and hippocampal dendritic retraction in their offspring. An adolescent-enriched environment experience prevented the behavior and morphologic changes in their offspring through hippocampal IGF-2 signaling. IGF-2 and an enriched environment may be a potential intervention to prevention of anxiety and brain atrophy in the offspring of parental opioid exposure.

Neuropsychopharmacology (20I4) 39, 2777-2787; doi:I0. I038/npp.20I4.128; published online 9 July 2014
\end{abstract}

\section{INTRODUCTION}

Opioid addiction, whether to opiates such as heroin and morphine or to non-medical use of opioids, is a major problem worldwide. Opioid abuse may be due to the illegal use of opiates or of illicit prescription drugs for the longterm treatment of chronic pain (Manchikanti et al, 2010). Chronic use of morphine can lead to brain structure and behavioral changes in individuals. Not only does long-term use of opioids have a negative effect on the individual but the parental addiction can also severely impact their offspring. Prenatal opioid exposure renders behavioral

\footnotetext{
*Correspondence: Dr R-P Dai, Department of Anesthesiology, The Second Xiangya Hospital of Central South University, Ren-Min Road No. 86, Changsha, Hunan Province 410011, China, Tel: +86 731 85295970, Fax: +86 731 852921 I5, E-mail: xyeyyrupingdai@gmail.com Received 6 February 2014; revised 20 April 2014; accepted 9 May 20I4; accepted article preview online 3 June 2014
}

changes in the offspring, such as addiction vulnerability and depression-like behavior (Klausz et al, 2011). In addition, prenatal opiate exposure also results in brain neurochemical changes, including dendritic retraction and the downregulation of dopamine receptor gene in adult offspring (DiNieri et al, 2011; Lu et al, 2012; Vathy, 2002).

Mounting evidence has shown that transgenerational effects of opioid exposure occur even in the absence of continuous use (for a review seeVassoler et al, 2014). Adolescent maternal morphine exposure, followed by a drug-free period of several weeks, still induced anxiety-like behavior and morphine sensitization in the offspring (Byrnes, 2005). Along with behavioral changes, adolescent maternal morphine exposure also altered the response of dopamine agonists and upregulated the dopamine- and opioid-related gene in the first and second generation (Byrnes et al, 2013). However, studies of transgenerational effects of adolescent morphine exposure have primarily involved adolescent female animals (Byrnes, 2005; Byrnes 
et al, 2011, 2013;Mei et al, 2009). The effects of adult opioid exposure on their offspring are unknown. Noticeably, the effects of morphine are quite distinct between adults and adolescents (Koek et al, 2012; Schramm-Sapyta et al, 2009; White et al, 2008). For example, adolescents are more sensitive to the rewarding effects of drugs and less sensitive to withdrawal effects (Schramm-Sapyta et al, 2009). Given that chronic use of opiates in the adult population has increased greatly in recent years, it is important to understand the effects of adult morphine exposure on their offspring (Compton and Volkow, 2006; Manchikanti et al, 2010)

It is well known that neurogenesis and dendritic plasticity are closely associated with the pathogenesis of anxiety and depression (Eisch and Petrik, 2012; Kheirbek and Hen, 2011; Mateus-Pinheiro et al, 2013). A few molecular signals linking dendritic plasticity and psychiatric disorders have been identified (Lanoue et al, 2013). Insulin-like growth factor (IGF-2) has been reported to regulate synapse formation, dendritic spine maturation, and memory consolidation (Agis-Balboa et al, 2011; Chen et al, 2011; Schmeisser et al, 2012). However, the molecular signals linking the effect of morphine exposure in parents to neuroplasticity and mood disorders in their offspring are still largely unknown.

Here, we showed that exposure to morphine in adult males and females led to two important effects in their adult male and female offspring: anxiety-like behaviors and reduced length and branch number of dendrites in the dentate gyrus (DG) of the hippocampus. In addition, IGF-2 in the hippocampus was downregulated in the offspring of parents exposed to morphine. Overexpression of IGF-2 by intra-DG injection of lentivirus encoding the human IGF-2 gene $\left(L V-I G F_{2}-E G F P\right)$ reversed the anxiety-like behavior. The anxiety-like behavior and the shortened dendrites in the hippocampus of adult offspring could be reversed by enriched environment (EE) exposure in the adolescent offspring. Finally, adolescent EE experience also prevented the downregulation of IGF-2 in the hippocampus. Thus, hippocampal IGF-2 has a critical role to link the neuropsychiatric disorders and retracted dendrites in the offspring of parents' morphine exposure, which can be modified by EE.

\section{MATERIALS AND METHODS}

\section{Subjects}

Sprague-Dawley rats of each sex (8 weeks of age) were purchased from Central South University Animal Services (Changsha, China). All animals in the study were group housed in light-controlled (1200-1200 h) and temperaturecontrolled $\left(21-24^{\circ} \mathrm{C}\right)$ rooms and were provided with food and water ad libitum. The experimental protocol was approved by the Animal Care and Use Committee of Central South University and conformed to the National Institutes of Health Guide for the Care and Use of Laboratory Animals.

\section{Adult Morphine Exposure}

Beginning at 8 weeks of age, female (defined as maternal), male (defined as paternal), or both (defined as biparental) were treated with morphine (morphine sulfate; Shenyang First Pharmaceutical, Shenyang, China) for a total of 10 days, using an increasing dose regimen, as described previously (Byrnes et al, 2011). On day 1 of exposure, animals received $2.5 \mathrm{mg} / \mathrm{kg}$ of morphine sulfate (subcutaneously) two times daily (0800 hours and 1600 hours). On each of the following 9 days, the dose of morphine was increased by $2.5 \mathrm{mg} / \mathrm{kg}$ each day, such that by the final day of treatment, rats received two times daily $25 \mathrm{mg} / \mathrm{kg}$ injections. Age-matched control animals received the saline vehicle $(0.9 \% \mathrm{NaCl}$, subcutaneously) two times daily with volumes adjusted to match those of drug-treated animals. Following this regimen, animals remained undisturbed in the colony until mating.

\section{Mating and Postpartum Care}

Four groups were arranged for mating and further study: male morphine exposure, female morphine exposure, both morphine exposure, and control (no morphine exposure). Twenty-one days after the final drug or vehicle administration (87 days of age), the morphine-exposed females or males were mated with the colony normal males or females, respectively, or were mated with each other. The salineinjected females and males were mated and served as controls. After mating, the females were monitored for pregnancy. The birth of each litter was designated postnatal day 0 (PND 0). On PND 1, pups were weighed and genders were determined. All litters were culled to four males and four females. Rat littermates of each sex were housed together throughout the experiment. Litters were weighed on PND 21 and PND 60. On PND 60, mortality rates in the control and morphine-exposed groups were determined from the number of offspring remaining divided by the total number of offspring on PND 0.

\section{Maternal Behavior Test}

Maternal behavior tests were performed in the postpartum female rats in the control and morphine-exposed groups as described previously (Zhao and Li, 2012). Detailed procedure has been described in the Supplementary Materials and Methods.

\section{IGF-2 Lentivirus Production}

High-titer lentivirus vectors encoding $I G F-2$ gene $\left(L V-I G F_{2^{-}}\right.$ EGFP) (pGV287-IGF 2 -EGFP, $2.2 \times 10^{9} \mathrm{U} / \mathrm{ml}$ ) or GFP only (lentivirus encoding enhanced green fluorescent protein gene $\left(L V\right.$-EGFP) (pGV287EGFP, $\left.1.8 \times 10^{9} \mathrm{U} / \mathrm{ml}\right)$ were obtained from Genenchem (Shanghai, China). The IGF-2 gene was ligased into pGV-287 plasmid (Genenchem, Shanghai, China) to generate pGV287-IGF-2. Production of LV-IGF $2^{-}$ EGFP or LV-EGFP is described in the Supplementary Materials and Methods.

\section{Bilateral Intra-DG Injection}

The method of intrainjection of lentivirus into the DG region of the bilateral hippocampus was described in our previous study with a slight modification (Dai et al, 2011). In brief, rats were anesthetized with an intraperitoneal injection of chloral hydrate $(40 \mathrm{mg} / \mathrm{kg})$, and securely placed into a stereotaxic device with bregma and lambda 
at a horizontal level. Two 30-G stainless-steel cannulae with 33-G stainless-steel stylet plugs were bilaterally implanted $0.5 \mathrm{~mm}$ above the DG regions of the hippocampus: anteroposterior $-3.50 \mathrm{~mm}$, relative to bregma; lateral $\pm 1.50 \mathrm{~mm}$; and dorsoventral $3.5 \mathrm{~mm}$ from skull, according to the atlas of Paxinos and Watson (1998).

\section{Behavioral Tests}

Elevated plus-maze test. Anxiety-like behavior was tested using the elevated plus-maze (EPM) test, as described previously (Weaver et al, 2006). Briefly, rats were placed in the middle of EPM apparatus, and their behavior was recorded by video camera for $5 \mathrm{~min}$. As an index of the anxiety-like behavior, data were expressed as the percentage of time spent in the open arms and the percentage of openarm entries, and as an index of overall locomotor activity, a total number of open- and closed-arm entries. The maze was cleaned after each trial.

Open-field test. The open-field test (OFT) was undertaken as described previously (Weaver et al, 2006). Briefly, animals were placed directly in one corner of the open field $(120 \mathrm{~cm} \times 120 \mathrm{~cm})$, which was divided into an $8 \times 8$ grid of squares. Movement of each animal in the area was video-recorded during the 5-min testing session. An observer blind to the experimental conditions analyzed the movement from the video recordings. Exploration was defined as the time spent in the inner $6 \times 6$ squares, and overall activity was defined as the number of squares crossed during the testing session.

Morris water-maze test. Morris water-maze test was undertaken as described previously (Li et al, 2008). Detailed information has been depicted in the Supplementary Materials and Methods.

\section{Adolescent EE Experience}

After weaning, the offspring from the groups that experienced $\mathrm{EE}$ were placed in a large vitrine $(60 \mathrm{~cm} \times 60 \mathrm{~cm} \times 40$ $\mathrm{cm}$ ), as a cohort of 12 , for $4 \mathrm{~h}$ every day. Inside the vitrine, there was approximately $2 \mathrm{~cm}$ of wood shavings covering the base, and toys (eg, wheels, ladders, plastic tunnels, wooden shelters, mazes made of colored building blocks, swings, wheel-runners) that were rotated every 2 days. Offspring from the control group (no EE experience) stayed in their regular home cages during this time. During nonexperimental hours, littermates from the $\mathrm{EE}$ condition groups were returned to their regular home cage and housed together. Littermates without EE condition were kept in home cage and housed together throughout the experiments.

\section{Golgi Staining and Quantitative Analysis of Dendrites and Spine}

Brains tissues from five rats from each group were processed for Golgi staining as described previously by us and others (Li et al, 2008; Wallace et al, 2006). Detailed experimental procedure has been described in the Supplementary Methods. Four to six granule cells within the DG per animal were imaged and traced for the analysis of dendritic length and branching. We selected for tracing only well-impregnated cells with soma located in the middle plane of the section, without broken arbors and no overlap with neighboring cells. Neurodraw and Image Prosoftware (MicroBrightField, Williston, VT, USA) were used for all analyses. Experimenters who carried out the analyses were blind to group identities. To determine dendritic spine density, spines from each of the neurons selected were counted on two $20 \mu \mathrm{m}$ unbranched dendrite segments $120 \mu \mathrm{m}$ away from the cell body at high power ( $\times 1000$ magnification) and data were expressed as the number of spines per $\mu \mathrm{m}$ of dendrite length. For each animal, cell values were averaged to derive a single value per variable (total dendritic length, number of branching points, and spine density).

\section{Immunohistochemistry}

IGF-2 immunohistochemistry was performed as described in our previous study (Dai et al, 2011). Briefly, transverse sections of the brain $(30 \mu \mathrm{m})$ were cut with a cryostat and mounted on 3-aminopropyl triethoxy-silane-coated slides, and rabbit anti-IGF $\mathrm{IG}_{2}$ antibodies (dilution 1:200; Abcam, Eugene, OR). The number of IGF-2-positive neurons was measured by an author blind with respect to the treatments.

\section{Western Blot}

Western blot analysis was performed as described by our previous study with a slight modification (Dai et al, 2011). Briefly, frozen hippocampus tissues were homogenized in a lysis buffer containing protease inhibitors cocktail (Roche, Mannheim, Germany) and PMSF (Sigma, St Louis, MO, USA). Samples were then centrifuged at 10000 r.p.m. for $15 \mathrm{~min}$ at $4{ }^{\circ} \mathrm{C}$. The supernatants were used for western blotting for IGF-2 with rabbit anti-IGF-2 (Abcam).

\section{RNA Extraction, Reverse Transcription and Real-Time PCR}

Total RNA was isolated from the hippocampus using TRIzol reagent based on the company protocol (Invitrogen, Carlsbad, CA, USA). Real-time PCR assay protocol was performed using SYBR Green Real-Time PCR Master Mixes (Roche) as described by our previous studies (Ruan et al, 2012). PCR primers were $5^{\prime}$-GCCGACAGGATGCAGAAGG AGATCA- $3^{\prime}$ and $5^{\prime}$-AAGCATTTGCGGTGGACGATGGA- $3^{\prime}$ ( $\beta$-actin); $5^{\prime}$-AGGGGAGCTTGTTGACACG- $3^{\prime}$ and $5^{\prime}$-GGGT ATCTGGGGAAGTCGTC-3' (IGF-2); 5'-ACCAGAGACCCTT TGCGGGGCT- $3^{\prime}$; and $5^{\prime}$-AAGTGTACTTCCTTCTGAGTCT- $3^{\prime}$ (IGF-1). The specific genes were normalized to the level of $\beta$-actin in each individual sample.

\section{Statistical Analysis}

Statistical analysis was performed using software SPSS 13.0 (SPSS, Chicago, IL) or GraphPad Prism 5 (GraphPad Software, San Diego, CA, USA). Data are presented as means \pm SEM. Differences between groups were compared using repeated-measures one-way ANOVA, followed by post hoc Bonferroni multiple comparison tests, where appropriate. Unpaired two-tailed Student's -test was used 
if only two groups were applied. Unless otherwise specified, those were the statistical analyses used in all the sections reported below. We considered differences to be significant if probability values were $\leqslant 0.05$.

\section{RESULTS}

There were no significant differences apparent in weight and ratio of gender among all offspring of the control and morphine-exposed groups in the indicated times measured. No significant differences in mortality rate on PND 60 were observed among the offspring of the control and morphineexposed groups (Supplementary Table S1).

\section{Development of Anxiety-Like Behaviors in the Adult Offspring of Morphine Exposure}

As Figure 1a shows, parental morphine exposure reduced the time spent in the open arm of the EPM in the male offspring, $\mathrm{F}(3,88)=12.51, p<0.0001$, and female offspring, $\mathrm{F}(3,84)=12.56, p<0.0001$. Post hoc tests showed that time spent in the open arms in the male or female offspring of morphine-exposed parents was significantly lower than for the corresponding control groups (male offspring, $p=0.009,0.001$, and 0.004 , respectively, for the paternal, maternal, or biparental morphine-exposed groups $v s$ the control groups; in female offspring, $p=0.004,0.006$, and
0.004, respectively, for paternal, maternal, or biparental groups $v s$ the corresponding control groups). Similarly, a significant difference in the number of open-arm entries was also observed in the male, $\mathrm{F}(3,58)=5.741, p<0.01$, and female offspring, $\mathrm{F}(3,58)=3.662, p<0.01$, of morphine-exposed groups. Post hoc tests shows the significantly less number of open-arm entries in the offspring of morphine-exposed groups than that of control group (male offspring, $p=0.026, p=0.029$ and $p=0.034$, respectively, for the paternal, maternal or bi-parental morphineexposed groups $v s$ the control groups; in female offspring, $p=0.034,0.026$, and 0.036 , respectively, for paternal, maternal, or biparental groups $v s$ the corresponding control groups)

OFT showed that the time spent in the inner area was markedly reduced in the adult offspring of the parents exposed to morphine, as compared with the offspring of the control parent groups, $\mathrm{F}(3,88)=29.371, p<0.001$ for male offspring, and $\mathrm{F}(3,83)=26.92, p<0.001$ for female offspring. There were no significant differences in the total travel distances between the offspring of morphine-exposed groups and their corresponding control offspring, $\mathrm{F}<1.00$ for both male and female groups.

There was no significant difference in the latency to find the platform in the adult offspring of morphine-exposed groups as compared with those of the corresponding control groups in the Morris water-maze test, $\mathrm{F}<1.00$ for
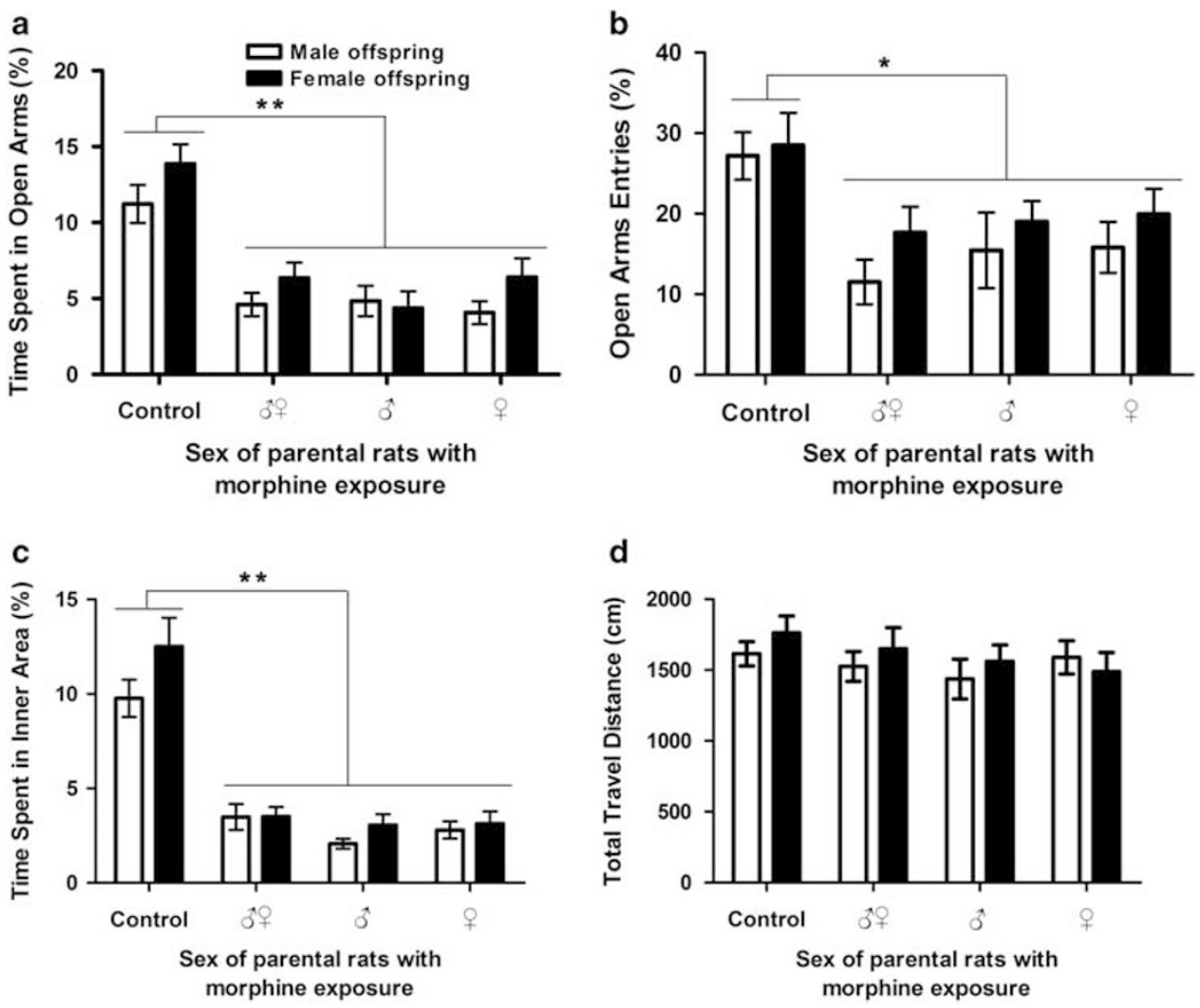

Figure I Anxiety-like behaviors in the elevated plus-maze (EPM) and open-field test (OFT) in the offspring of morphine-exposed groups. ( $a$ and b) Quantitative analysis of the time spent in the open arm (a) and the percentage of open arm entries (b) in EPM in both male and female offspring of the morphine-exposed groups. (c) Reduced time spent in the inner area in the offspring of morphine-exposed groups in OFT. (d) Quantitative analysis of total travel distance among offspring of the control and morphine-exposed groups. Values are expressed as a mean $\pm S E M$. $* P<0.05$ and $* * p<0.00 I$, respectively, vs offspring of the control group. 
both male and female groups (Supplementary Figure S1). Thus, parental morphine exposure mainly resulted in the anxious phenotype, but did not impair the learning and memory in their adult offspring.

\section{Effects of Morphine Exposure on Dendritic}

Morphogenesis in the DG of the Hippocampus in their Adult Offspring

Given that synaptic plasticity is implicated in the pathogenesis of anxiety and depression (Bessa et al, 2009; Kheirbek and Hen, 2011), we performed Golgi staining to examine dendritic morphology in the DG (Figure 2). Both in the male and female offspring of the morphine-exposed groups, the dendritic length (Figure 2e) were greatly reduced as compared with the corresponding offspring of the control groups $(\mathrm{F}(3,16)=24.08, p<0.001, \mathrm{~F}(3,16)=9.009$, $p<0.001$, respectively, for male and female offspring). Dendritic branching number (Figure 2f) was also reduced
$(\mathrm{F}(2,16)=3.906, p=0.029, \mathrm{~F}(3,16)=5.714, p=0.007$, respectively, for male and female offspring). No significant differences were observed in the spine density among the offspring of all groups (Figure 2g). Post hoc analyses showed that, within the offspring of morphine-exposed fathers, mothers, or both parents, there were no significant differences in dendritic length, branch points, or spine density when compared with each other. These findings indicate that parents' exposure to morphine-whether maternal, paternal, or biparental-led to the dendritic retraction in both their male and female offspring.

As the behavioral and hippocampal morphologic changes in the offspring of parental morphine exposure may be due to the maternal care alterations (Kikusui and Mori, 2009), we evaluated maternal behaviors on postpartum days 6-8, as described previously (Zhao and $\mathrm{Li}, 2012$ ). As shown in Supplementary Figure S2, among the different groups with or without parental morphine exposure, there were no significant differences in the duration of crouching
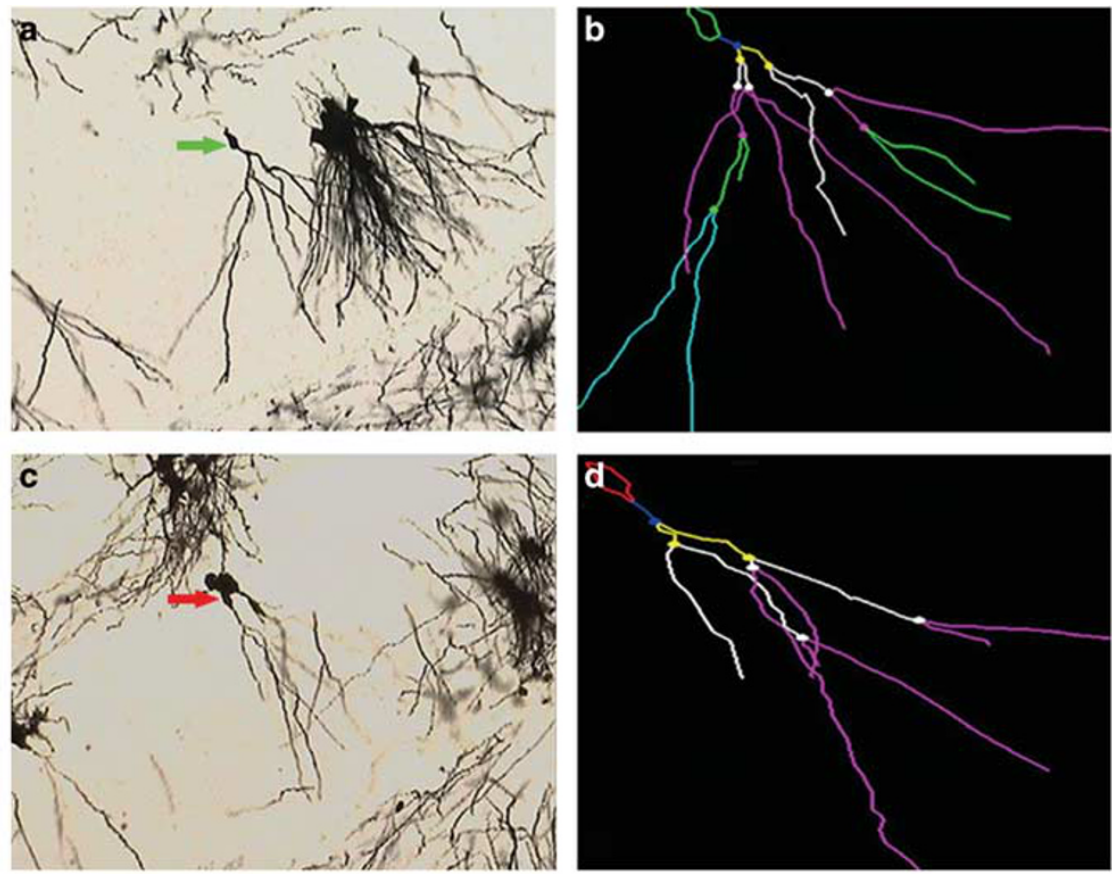

e

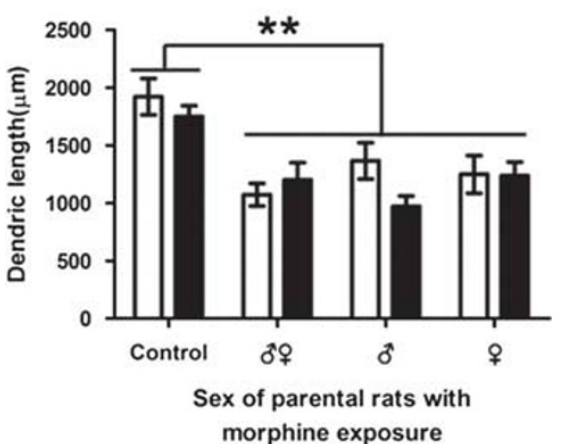

f

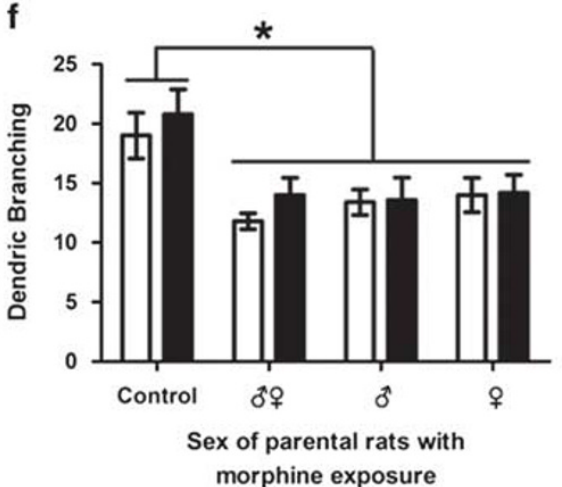

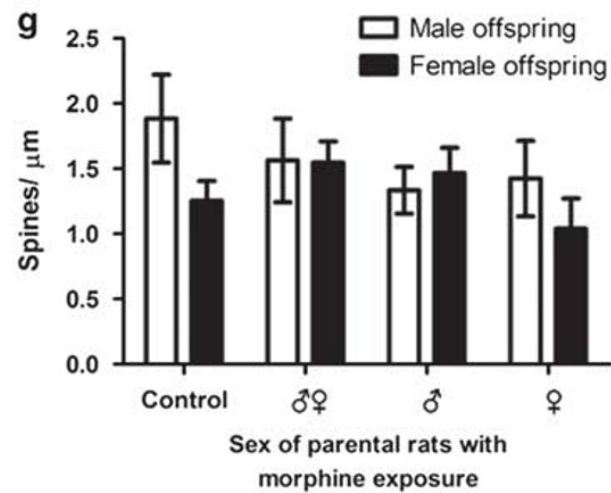

Figure 2 Effect of adult morphine exposure on dendritic morphogenesis in the dentate gyrus (DG) of the hippocampus in their offspring. ( $a$ and b) Representative photomicrographs (a) and its reconstruction (b) of Golgi staining in the subgranular zone of the DG. Arrow indicates the cell body of neuron. (c-e) Quantitative analysis of dendritic length (c), number of branching points ( $f$ ) and spine density, as indicated by the number of spine per $\mu \mathrm{m}(\mathrm{g})$. Values are expressed as a mean \pm SEM. $* P<0.05$ and ${ }^{*} * 0<0.00 \mathrm{I}$, respectively, vs offspring of the control group. 


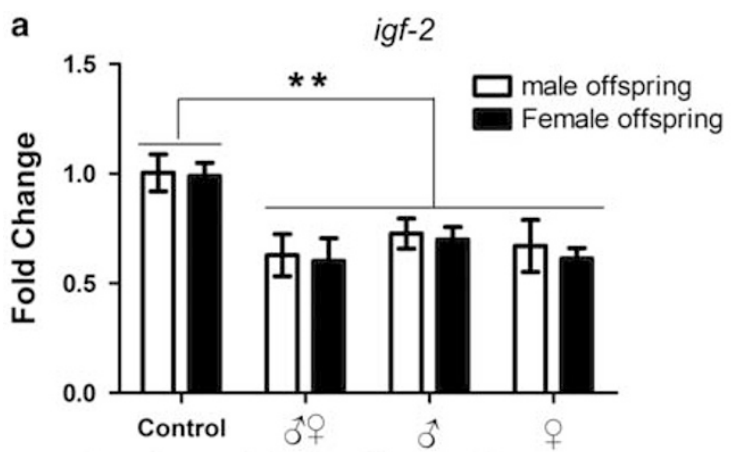

Sex of parental rats with morphine exposure

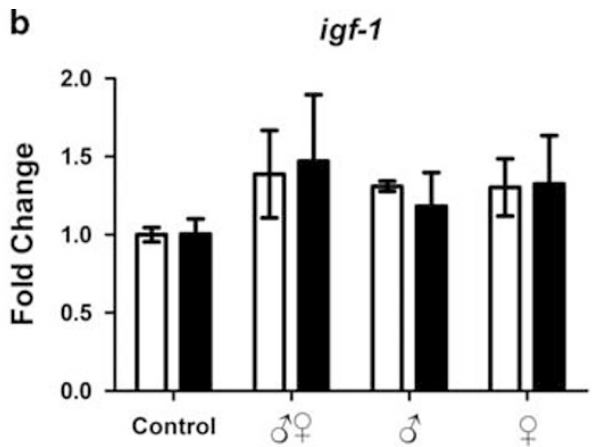

Sex of parental rats with morphine exposure

C
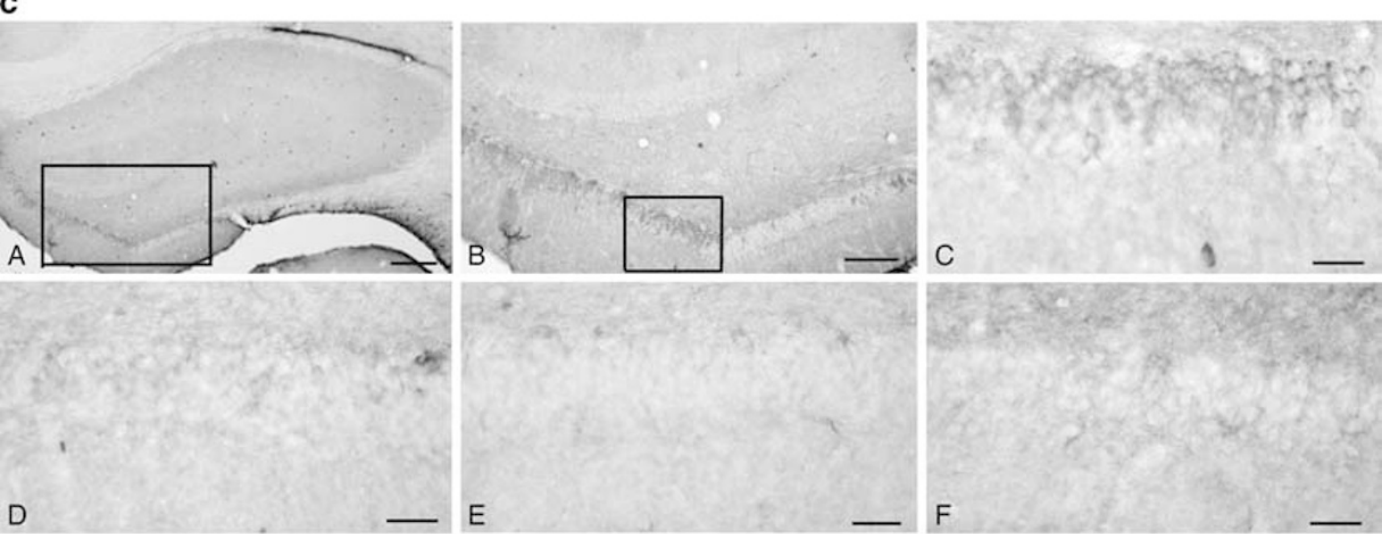

D
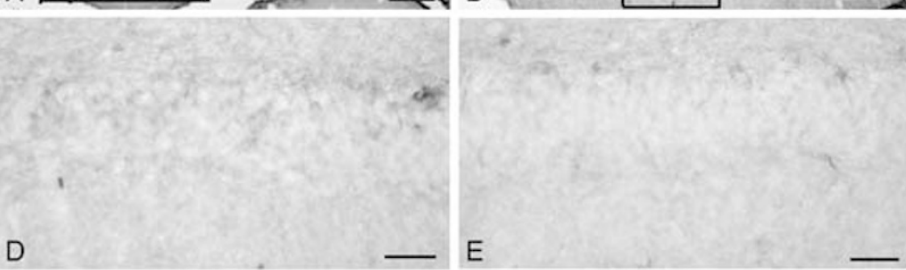

d

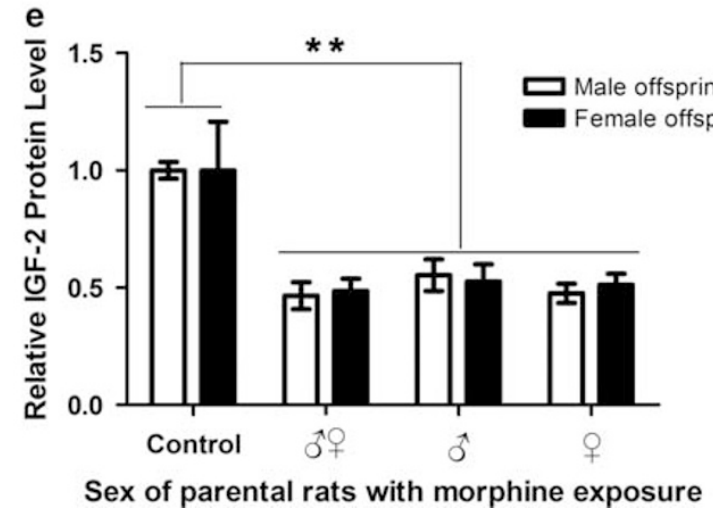

Figure 3 Downregulation of hippocampal insulin growth factor-2 (IGF-2) in the offspring of adult morphine-exposed groups. (a and b) Quantitative analysis of IGF-2 (a) and IGF-I (b) mRNA levels in the hippocampus in the offspring of the control or morphine-exposed groups. (c) IGF-2 immunohistochemistry in the hippocampus. (A) representative photomicrographs of IGF-2 immunohistochemical staining in the offspring of the control group; (B) higher magnification of inset in (A); (C) higher magnification of inset in (B); (D-F) representative photomicrograph of IGF-2 expression in the offspring of adult male (D), female (E), and both (F) morphine exposure. Bar, I00 $\mu \mathrm{m}$. (d) Representative western blot image of IGF-2 in the hippocampus. (e) Semiquantitative analysis of IGF-2 protein expression in the offspring of morphine-exposed or the control groups. Values are expressed as a mean \pm SEM. $* P<0.05$ and ${ }^{*} * p<0.001$, respectively, vs offspring of the control group.

Figure 4 Effects of overexpression of insulin growth factor-2 (IGF-2) in the adolescent offspring of morphine-exposed groups on anxiety-like behavior in their adulthood ( $\mathrm{a}$ and $\mathrm{b}$ ) Representative photomicrographs of Hoechst (blue, staining nuclei) and green fluorescent protein (GFP) expression (green) in the dentate gyrus (DG) after bilateral injection of LV-IGF 2 -EGFP or LV-EGFP. (c and d) Quantitative analysis of IGF-2 gene expression in adult male (c) and female (d) offspring receiving LV-IGF - EGFP or LV-EGFP injection in adolescence. (e-g) Representative western blot image (e) and its semiquantitative analysis of IGF-2 expression in male (f) and female (g) offspring after LV-IGF 2 -EGFP or LV-EGFP injection. (h and i) Quantitative analysis of the time spent in the open arm in male $(\mathrm{h})$ and female (i) offspring of morphine-exposed groups after LV-EGF 2 -EGFP or LV-EGFP injection. ( $\mathrm{k}$ and $\mathrm{k}$ ) Quantitative analysis of time spent inner area in the male $(j)$ or female ( $k$ ) offspring received LV-IGF 2 -EGFP or LV-EGFP bilateral DG injection. Values are expressed as a mean $\pm \mathrm{SEM}$. $* P<0.05$ and $* * * 0.00 \mathrm{I}$, respectively, rats received LV-IGF-2 injection vs the responding littermate received LV-EGFP injection; ${ }^{\# \#} p<0.01$ and ${ }^{\# \# \#} p<0.00$ I, offspring of the morphine-exposed group vs offspring of the control group. LV-IGF 2 -EGFP: lentivirus encoding IGF-2 gene; LV-EGFP: lentivirus delivered EGFP gene, the reporter gene. 
$((\mathrm{F} \quad(3,15)=0.078, p>0.05)$, duration of pup retrieval ( $(\mathrm{F}(3,15)=0.177, p>0.05)$, duration of pup licking $(\mathrm{F}(3,15)=0.508, p>0.05)$, and duration of nest building
(F $(3,15)=0.038, p>0.05)$. Thus, the anxious phenotype of the offspring of morphine-exposed parents is unlikely because of the inadequate maternal care.

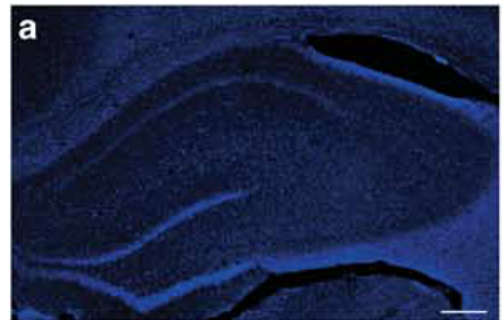

C

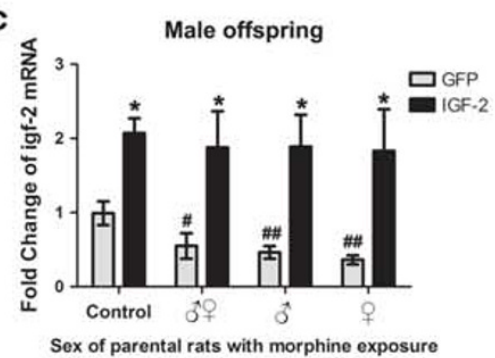

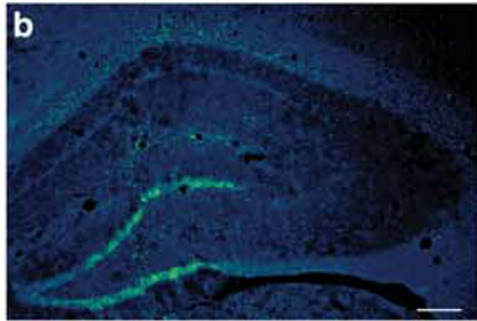

d

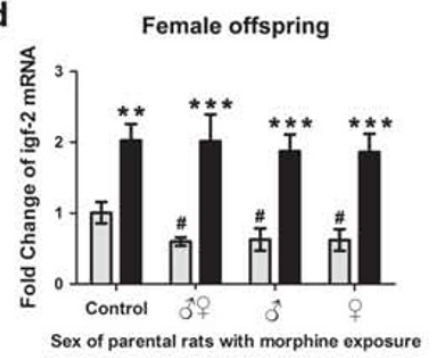

e

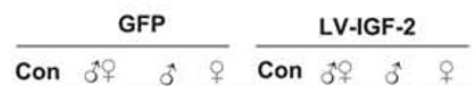

Male offspring

IGF-2

GAPDH

Female offspring

IGF-2

GAPDH

f

Male offspring

g

Female offspring

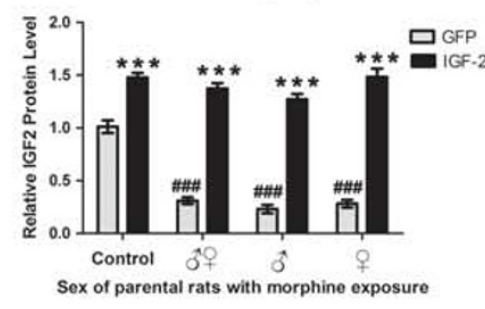

h
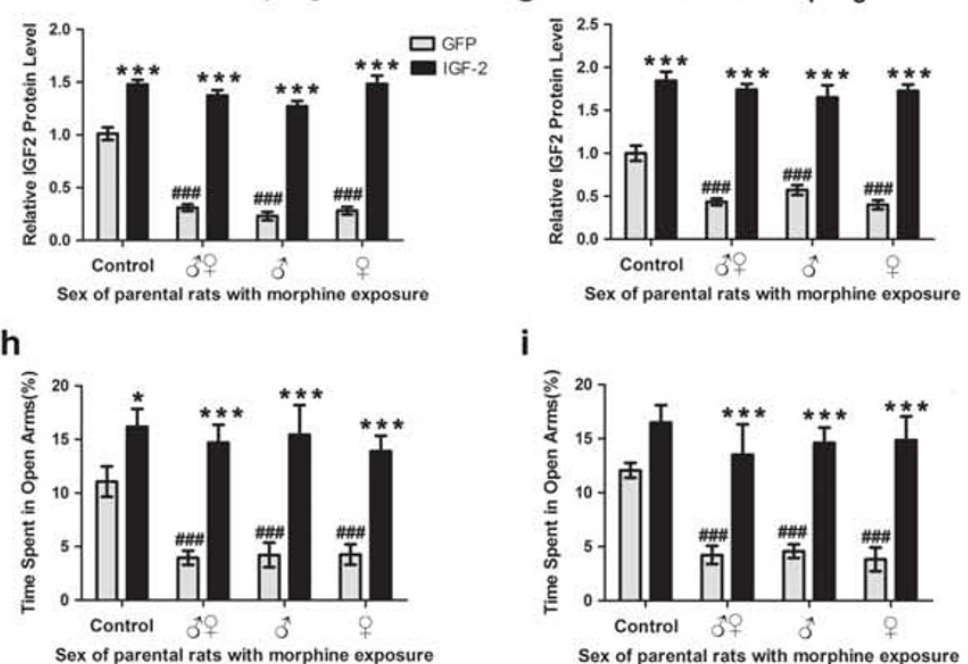

i

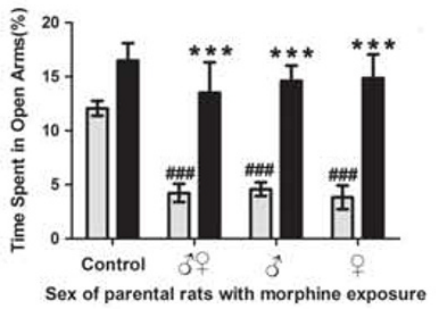

j

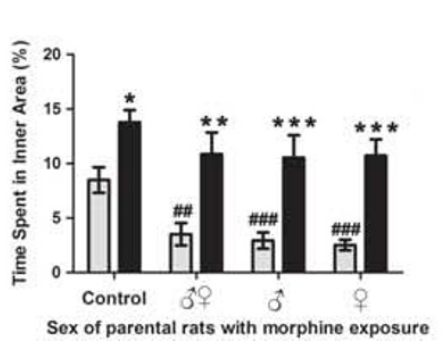

k

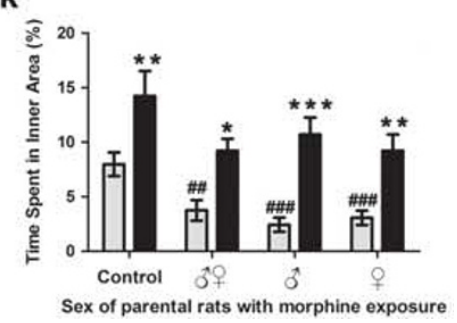


Effect of the Morphine Exposure on the Hippocampal IGF-2 Expression in their Adult Offspring

IGF-2 has recently been reported to be involved in dendritic plasticity and memory consolidation and enhancement (Agis-Balboa et al, 2011; Chen et al, 2011; Schmeisser et al, 2012). Thus, we hypothesized that the dendritic retraction and anxiety-like behavior may be closely associated with IGF-2 signaling. As shown in Figure 3a, IGF-2 mRNA markedly decreased in the offspring of paternal, maternal, and parental morphine-exposed groups, as compared with those of the control groups. However, the IGF-1 gene was not altered in the offspring of the parents with morphine exposure (Figure $3 \mathrm{~b}$ ). Immunohistochemistry and western blot also showed that IGF-2 expressed in the neurons was significantly decreased in the offspring of parents with morphine exposure compared with controls (Figures $3 c$ and $d$ ). These findings suggest the downregulation of IGF-2 in the DG region in the offspring of morphine-exposed parents.

\section{Overexpression of IGF-2 in the Adolescent Period Prevented Anxiety in the Adult Offspring}

To further investigate whether downregulation of IGF-2 in the hippocampus contributed to anxiety-like behavior and dendritic shortening, we constructed the LV-EGFP (control) or $L V-I G F_{2}-E G F P$ for IGF-2 overexpression. LV-IGF - -EGFP or LV-EGFP was bilaterally injected into the DG region of the hippocampus in the male and female offspring of morphine-exposed parents on PND 28, a critical period of adolescence. On PND 60, the injected rats undertook the behavior tests, followed by biochemical examination. As shown in Figure 4b, EGFP was highly expressed in the injection zone at 32 days after injection, indicating successful overexpression of the target gene. Consistently, IGF-2 mRNA and protein were also highly expressed in the hippocampus after bilateral injection of LV-IGF 2 -EGFP (Figures $4 \mathrm{c}-\mathrm{g}$ ).

Rats overexpressing IGF-2 in the hippocampus exhibited more time spent in the open-arm (Figures $4 \mathrm{~h}$ and i) $(\mathrm{F}(3,54)=32.73, p<0.01$ in male groups, $\mathrm{F}(3,56)=35.58$, $p<0.01$ in female groups) and more time spent in the inner area (Figures $4 \mathrm{j}$ and $\mathrm{k})(\mathrm{F}(3,54)=27.86, p<0.01$ in male groups, $\mathrm{F}(3,56)=31.12, p<0.01$ in female groups) as compared with the littermates injected by LV-EGFP. This indicates that IGF-2 overexpression could prevent the development of anxiety-like behaviors in the offspring of rats with morphine exposure.

\section{Effect of Adolescent EE on Anxiety and Hippocampal IGF-2 Expression in the Offspring}

Early life environmental enrichment (EE) experience reduces the anxious phenotype in the rats exposed to stressors (Baldini et al, 2013; Francis et al, 2002). Therefore, we attempted to examine whether adolescent $\mathrm{EE}$ exposure could reverse the adult anxiety-like behavior in the offspring of parent animals exposed to morphine.

As shown in Figures $5 \mathrm{a}$ and $\mathrm{b}$, in male and female offspring of different morphine-exposed groups, rats with adolescent EE experience exhibited more time spent in the EPM open arms than littermates without EE experience $(\mathrm{F}(3,102)=49.07, p<0.01$ in male groups, $\mathrm{F}(3,103)=31.08$, $p<0.01$ in female groups). In the offspring of the control group, rats with adolescent EE experience did not increase the time spent in EPM open arms ( $p>0.05$ for both male and female offspring). Similarly, rats that received adolescent EE experience spent more time in the inner area of the open field than littermates without EE (Figures $5 c$ and d). Thus, adolescent EE experience appeared to prevent the occurrence of anxiety-like behaviors in the offspring of rats with morphine exposure.

In the offspring of rats with morphine exposure, the length and branching points in dendrites of neurons in the DG region of the hippocampus were less than the offspring of the control group (Figures $2 \mathrm{~b}$ and $\mathrm{c}$ ). However, among the adult offspring with adolescent $\mathrm{EE}$ experience, there was no significant difference in dendritic parameters between the morphine-exposed groups and the control group (Supplementary Figure S3). These findings suggest that adolescent EE experience could normalize the dendritic retraction in the offspring of parental morphine exposure.

As the downregulation of hippocampal IGF-2 contributed to anxious phenotype in the offspring of morphine-exposed groups, we speculated that IGF-2 signaling might mediate the preventive effect of adolescent EE on anxiety. Indeed, adolescent EE could reverse the downregulation of IGF-2 in the hippocampus in the male (Figures $5 \mathrm{~g}$ and $\mathrm{h}$ ) and female offspring (Figures $5 \mathrm{~g}$ and $\mathrm{i}$ ). These findings support the hypothesis that adolescent EE reduces the anxious phenotype through hippocampal IGF-2 signaling.

\section{DISCUSSION}

Extensive studies have shown that adolescent maternal morphine exposure can lead to anxiety-like behavior in their female offspring (for a review see Vassoler et al, 2014). In male rats, adolescent morphine exposure delayed sexual maturation, resulted in smaller litters, and induced significant endocrine modification in their offspring as adults (Cicero et al, 1991). In the present study, adult morphine exposure did not affect general parameters perhaps because the animals had reached full sexual maturity when the drug was administered. Previous studies showed sex-differential behavior in the offspring of adolescent morphine-exposed rats (Byrnes, 2005; Johnson et al, 2011). Female offspring developed anxiety-like behavior, whereas male offspring demonstrated significantly enhanced morphine sensitization (Byrnes, 2005; Johnson et al, 2011). By contrast, in our study, both male and female offspring demonstrated anxiety-like behavior in the EPM and OFT procedures. The discrepant observations of anxiety-like behavior in the present study compared with previous studies are likely due to age-related differences in the effect of morphine exposure. Indeed, adolescence represents a period of significant brain maturation consisting of behavioral characteristics, cortex myelin accretion, and drug vulnerability (Chen et al, 2009; Mengler et al, 2014; Spear, 2000). In contrast, in the adult with the endocrinal and brain maturity, the sexdifferential response of offspring may not be obvious. Supporting this hypothesis, a previous electrophysiologic study showed that long-term potentiation in the hippocampus was impaired in both male and female offspring of morphine-exposed mothers, fathers, and both parents 
a

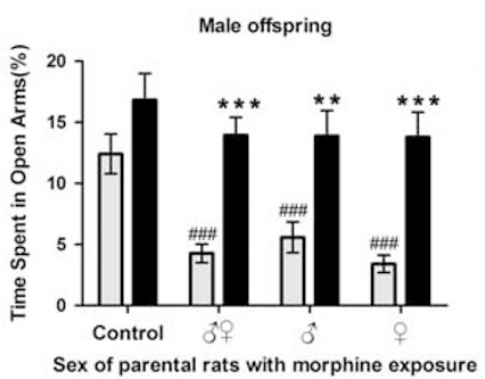

C

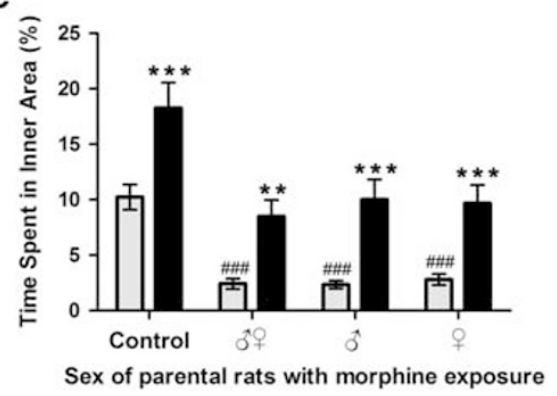

b
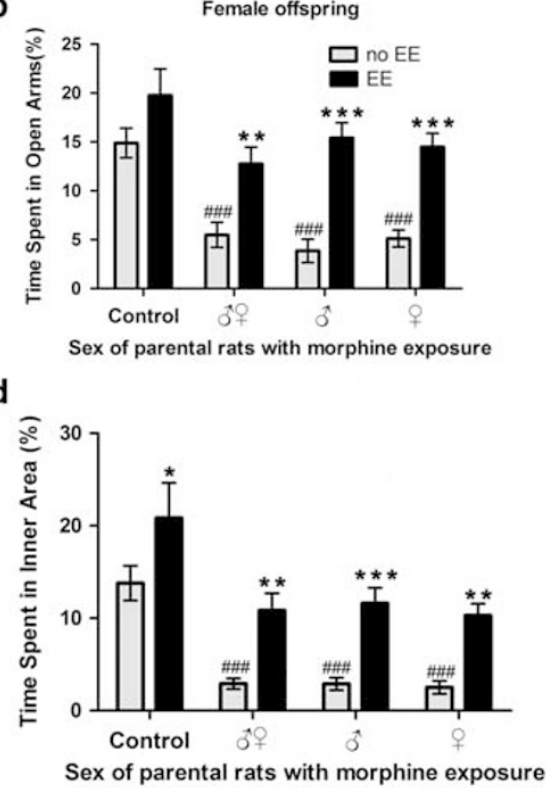

e

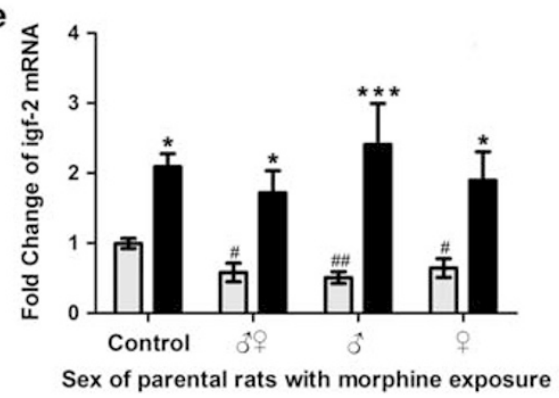

f

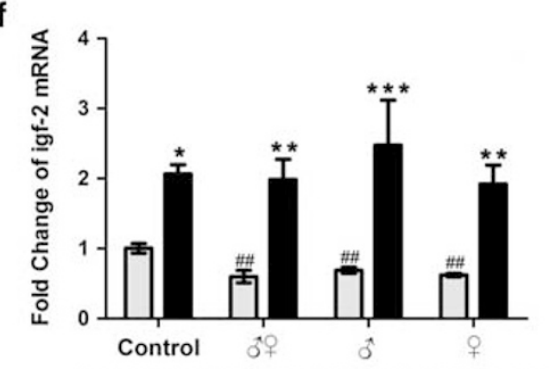

Sex of parental rats with morphine exposure

g
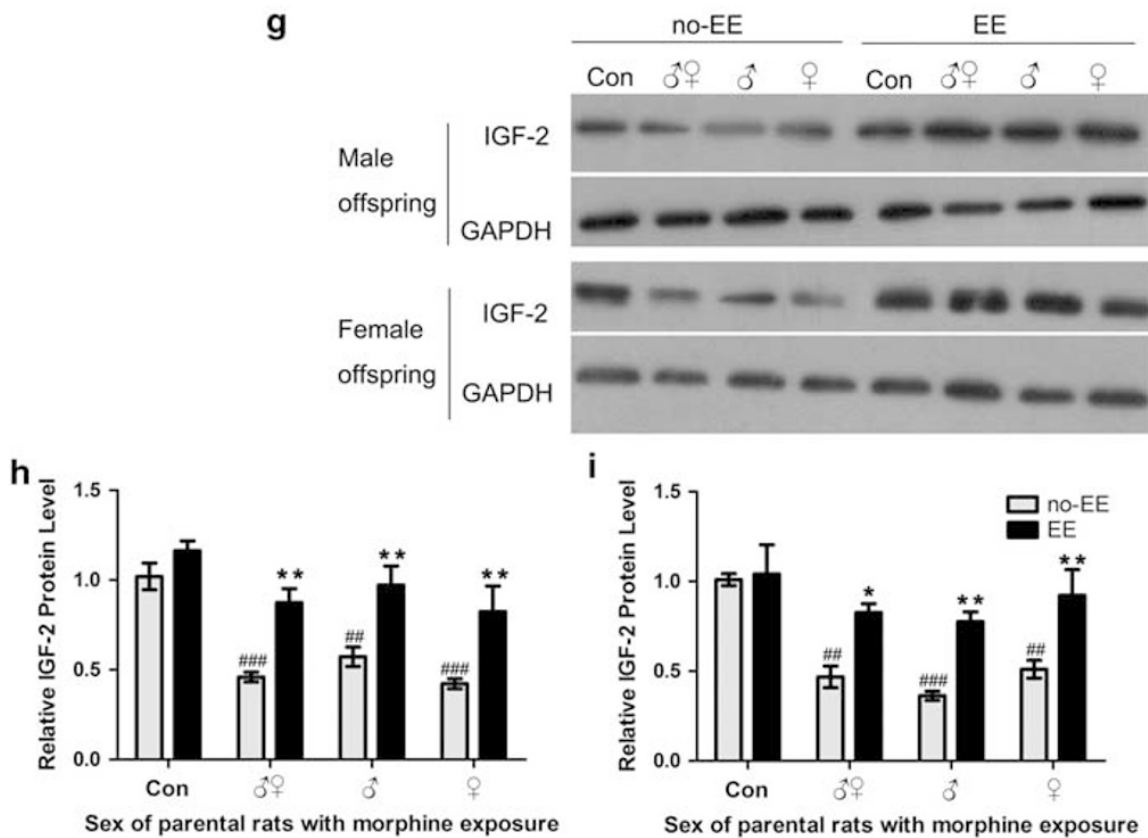

Figure 5 Anxiety-like responses in rats receiving adolescent environmental enrichment (EE), in relation to hippocampal insulin growth factor-2 (IGF-2) signaling. (a-d) Quantitative analysis of the time spent in open arm ( $a$ and b) in elevated plus-maze experiment, and the time spent in the open arm (c and d) in open-field test in the adolescent offspring receiving EE or the responding littermate without EE experience. (e and f) Real-time PCR showing IGF-2 gene expression in the male (e) and female ( $f$ ) offspring with or without EE experience. ( $g-i)$ Western blot of IGF-2 (g) and its semiquantitative analysis in IGF-2 protein expression in the male (h) and female (i) offspring with or without EE experience. Values are expressed as a mean $\pm S E M$. * $P<<0.05$ and $* * *<<0.001$, respectively, rats received EE experience vs the responding littermate without EE experience; $\# p<0.01$ and \#\#\# $p<0.001$, offspring of the morphineexposed group vs offspring of the control group. 
(Sarkaki et al, 2008). Further study may be warranted to investigate the age-dependent effect of morphine-exposed animals on their offspring.

Impaired maternal care during the early developmental period could lead to behavioral and neuroanatomical changes in offspring at adulthood (Kikusui and Mori, 2009). In the present study, classic maternal behaviorsincluding duration of crouching, licking, retrieving pups, and nest building-were not significantly different among the control and morphine-exposed groups. In addition, paternal morphine exposure alone also brought out the anxiety-like behavior in the offspring. Thus, the anxious phenotype and dendritic retraction in the adult offspring of morphine-exposed mothers, fathers, and both parents were unlikely because of inadequate maternal care in the early developmental period.

Dendritic and synaptic plasticity is implicated in the pathogenesis of anxiety and depression (Eisch and Petrik, 2012; Kheirbek and Hen, 2011). Concomitant with the anxious phenotype, we found reduced lengths and numbers of dendritic branches in DG neurons in the offspring of the morphine-exposed mothers, fathers, and both parents. Direct prenatal morphine exposure is known to reduce dendrite length and branch number in the adult offspring (Lu et al, 2012; Mei et al, 2009). However, the dendritic deficits in the animals from this study did not result from direct exposure to morphine, as the morphine treatments of their parents terminated several weeks before the initiation of mating. Thus, the intergenerational effects of morphine exposure may be mediated by other mechanisms such as epigenetic mechanisms or persistent stress (Czeh et al, 2001).

IGF-2 in the hippocampus has been reported to be involved in neurogenesis and dendritic plasticity, which are greatly inhibited in anxiety and depression (Bracko et al, 2012; Mateus-Pinheiro et al, 2013; Schmeisser et al, 2012). Although elevation of hippocampal IGF-2 is closely related to the reduced anxiety scores in stressed rats (Cline et al, 2012), the role of IGF-2 in the pathogenesis of anxiety is obscure. In the present study, hippocampal IGF-2 expression was downregulated, accompanied by the dendritic retraction in the offspring of morphine-exposed groups. Overexpression of IGF-2 in the hippocampus of the adolescent offspring prevented the anxiety-like behaviors in the adult. These findings suggest that downregulation of IGF- 2 contributes to the dendritic retraction and anxiety in the adult offspring of rats with morphine exposure. More interestingly, overexpression of IGF-2 in the hippocampus also reduced the anxious response in the offspring of control group. The antianxious response of IGF-2 in the normal rats may be due to the increased IGF-2 promotes dendritic morphogenesis in the hippocampus.

Early life EE could protect from, or remediate, the development of highly emotional and anxious responses in the rats exposed to stressors (Baldini et al, 2013; Francis et $a l, 2002)$. In the present study, adolescent EE evidently prevented the anxiety-like behaviors and dendritic retraction in the adult offspring of morphine-exposed rats, suggesting that early life EE can affect brain development and shape individual differences in anxiety and depression. However, the mechanisms underlying the effects of $\mathrm{EE}$ on anxiety and dendritic plasticity are not clear. One possibility is that $\mathrm{EE}$ may regulate neurotrophins which could couple the neuroplasticity and anxiety/depression. In the work reported here, IGF-2 can be upregulated by adolescent EE. These findings indicate that hippocampal IGF-2 has a critical role in linking the effects of adolescent EE with the brain plasticity and the antianxiety effect.

In conclusion, the present study has shown that adultmorphine exposure renders anxiety and hippocampal dendritic retraction in adult offspring. Hippocampal IGF-2 signaling may be a critical mediator that links neuropsychiatric disorders with the dendritic remodeling in the intergenerational effects of morphine exposure. The data indicate that adolescent EE experience prevented the behavioral and brain structural changes in the animal during adulthood, via hippocampal IGF-2 signaling. Given the high incidence of opiate exposure in adult humans, the potential for behavioral changes in their offspring should be taken as a serious public health concern. Timely intervention by EE or pharmacologic treatment targeting IGF-2 signaling may be simple and helpful methods to prevent psychological disorders in the offspring of chronic opiate users, thus potentially reducing the risk of serious social burden.

\section{FUNDING AND DISCLOSURE}

The author(s) declare that no financial support or compensation has been received from any individual or corporate entity over the past 3 years for research or professional services and there are no personal financial holdings that could be perceived as constituting a potential conflict of interest.

\section{ACKNOWLEDGEMENTS}

This study was supported by funding from the Program for New Century Excellent Talents in University (NCET-120543) and Hunan Provincial Natural Science Foundation (13JJ1007 to R Dai); and National Natural Science Foundation of China (81070897 to R Dai; 31171151 and 30971533 to C Li; and 81100908 to F Bi). We thank Ms Kate Rees from the University of South Australia for her critical reading of the manuscript. We thank LetPub (http://www.letpub.com) for its linguistic assistance during the preparation of this manuscript.

\section{REFERENCES}

Agis-Balboa RC, Arcos-Diaz D, Wittnam J, Govindarajan N, Blom $\mathrm{K}$, Burkhardt S et al (2011). A hippocampal insulin-growth factor 2 pathway regulates the extinction of fear memories. EMBO J 30: 4071-4083.

Baldini S, Restani L, Baroncelli L, Coltelli M, Franco R, Cenni MC et al (2013). Enriched early life experiences reduce adult anxietylike behavior in rats: a role for insulin-like growth factor 1 . J Neurosci 33: 11715-11723.

Bessa JM, Ferreira D, Melo I, Marques F, Cerqueira JJ, Palha JA et al (2009). The mood-improving actions of antidepressants do not depend on neurogenesis but are associated with neuronal remodeling. Mol Psychiatry 14: 764-773.

Bracko O, Singer T, Aigner S, Knobloch M, Winner B, Ray J et al (2012). Gene expression profiling of neural stem cells and their neuronal progeny reveals IGF2 as a regulator of adult hippocampal neurogenesis. J Neurosci 32: 3376-3387. 
Byrnes EM (2005). Transgenerational consequences of adolescent morphine exposure in female rats: effects on anxiety-like behaviors and morphine sensitization in adult offspring. Psychopharmacology (Berl) 182: 537-544.

Byrnes JJ, Babb JA, Scanlan VF, Byrnes EM (2011). Adolescent opioid exposure in female rats: transgenerational effects on morphine analgesia and anxiety-like behavior in adult offspring. Behav Brain Res 218: 200-205.

Byrnes JJ, Johnson NL, Carini LM, Byrnes EM (2013). Multigenerational effects of adolescent morphine exposure on dopamine D2 receptor function. Psychopharmacology (Berl) 227: 263-272.

Chen DY, Stern SA, Garcia-Osta A, Saunier-Rebori B, Pollonini G, Bambah-Mukku D et al (2011). A critical role for IGF-II in memory consolidation and enhancement. Nature 469: 491-497.

Chen CY, Storr CL, Anthony JC (2009). Early-onset drug use and risk for drug dependence problems. Addict Behav 34: 319-322.

Cline BH, Steinbusch HW, Malin D, Revishchin AV, Pavlova GV, Cespuglio $\mathrm{R}$ et al (2012). The neuronal insulin sensitizer dicholine succinate reduces stress-induced depressive traits and memory deficit: possible role of insulin-like growth factor 2. BMC Neurosci 13: 110.

Cicero TJ, Adams ML, Giordano A, Miller BT, O'Connor L, Nock B (1991). Influence of morphine exposure during adolescence on the sexual maturation of male rats and the development of their offspring. J Pharmacol Exp Ther 256: 1086-1093.

Compton WM, Volkow ND (2006). Abuse of prescription drugs and the risk of addiction. Drug Alcohol Depend 83(Suppl 1): S4-S7.

Czeh B, Michaelis T, Watanabe T, Frahm J, de Biurrun G, van Kampen $M$ et al (2001). Stress-induced changes in cerebral metabolites, hippocampal volume, and cell proliferation are prevented by antidepressant treatment with tianeptine. Proc Natl Acad Sci USA 98: 12796-12801.

Dai RP, Li CQ, Zhang JW, Li F, Shi XD, Zhang JY et al (2011). Biphasic activation of extracellular signal-regulated kinase in anterior cingulate cortex distinctly regulates the development of pain-related anxiety and mechanical hypersensitivity in rats after incision. Anesthesiology 115: 604-613.

DiNieri JA, Wang X, Szutorisz H, Spano SM, Kaur J, Casaccia P et al (2011). Maternal cannabis use alters ventral striatal dopamine D2 gene regulation in the offspring. Biol Psychiatry 70: 763-769.

Eisch AJ, Petrik D (2012). Depression and hippocampal neurogenesis: a road to remission. Science (New York, NY) 338: 72-75.

Francis DD, Diorio J, Plotsky PM, Meaney MJ (2002). Environmental enrichment reverses the effects of maternal separation on stress reactivity. J Neurosci 22: 7840-7843.

Johnson NL, Carini L, Schenk ME, Stewart M, Byrnes EM (2011). Adolescent opiate exposure in the female rat induces subtle alterations in maternal care and transgenerational effects on play behavior. Front Psychiatry 2: 29.

Kheirbek MA, Hen R (2011). Dorsal vs ventral hippocampal neurogenesis: implications for cognition and mood. Neuropsychopharmacology 36: 373-374.

Kikusui T, Mori Y (2009). Behavioral and neurochemical consequences of early weaning in rodents. J Neuroendocrinol 21: 427-431.

Klausz B, Pinter O, Sobor M, Gyarmati Z, Furst Z, Timar J et al (2011). Changes in adaptability following perinatal morphine exposure in juvenile and adult rats. Eur J Pharmacol 654: $166-172$.

Koek W, France CP, Javors MA (2012). Morphine-induced motor stimulation, motor incoordination, and hypothermia in adolescent and adult mice. Psychopharmacology (Berl) 219: 1027-1037.

Lanoue V, Usardi A, Sigoillot SM, Talleur M, Iyer K, Mariani J et al (2013). The adhesion-GPCR BAI3, a gene linked to psychiatric disorders, regulates dendrite morphogenesis in neurons. Mol Psychiatry 18: 943-950.

Li CQ, Liu D, Huang L, Wang H, Zhang JY, Luo XG (2008). Cytosine arabinoside treatment impairs the remote spatial memory function and induces dendritic retraction in the anterior cingulate cortex of rats. Brain Res Bull 77: 237-240.

Lu R, Liu X, Long H, Ma L (2012). Effects of prenatal cocaine and heroin exposure on neuronal dendrite morphogenesis and spatial recognition memory in mice. Neurosci Lett 522: 128-133.

Manchikanti L, Fellows B, Ailinani H, Pampati V (2010). Therapeutic use, abuse, and nonmedical use of opioids: a tenyear perspective. Pain Physician 13: 401-435.

Mateus-Pinheiro A, Patricio P, Bessa JM, Sousa N, Pinto L (2013). Cell genesis and dendritic plasticity: a neuroplastic pas de deux in the onset and remission from depression. Mol Psychiatry 18: $748-750$

Mengler L, Khmelinskii A, Diedenhofen M, Po C, Staring M, Lelieveldt BP et al (2014). Brain maturation of the adolescent rat cortex and striatum: changes in volume and myelination. NeuroImage 84: 35-44.

Mei B, Niu L, Cao B, Huang D, Zhou Y (2009). Prenatal morphine exposure alters the layer II/III pyramidal neurons morphology in lateral secondary visual cortex of juvenile rats. Synapse 63: $1154-1161$

Paxinos G, Watson C (1998). The Rat Brain in Stereotaxic Coordinates. Academic Press: New York, NY, USA.

Ruan W, Xu JM, Li SB, Yuan LQ, Dai RP (2012). Effects of downregulation of microRNA-23a on TNF-alpha-induced endothelial cell apoptosis through caspase-dependent pathways. Cardiovasc Res 93: 623-632.

Sarkaki A, Assaei R, Motamedi F, Badavi M, Pajouhi N (2008). Effect of parental morphine addiction on hippocampal longterm potentiation in rats offspring. Behav Brain Res 186: 72-77.

Schmeisser MJ, Baumann B, Johannsen S, Vindedal GF, Jensen V, Hvalby OC et al (2012). IkappaB kinase/nuclear factor kappaBdependent insulin-like growth factor 2 (Igf2) expression regulates synapse formation and spine maturation via Igf2 receptor signaling. J Neurosci 32: 5688-5703.

Schramm-Sapyta NL, Walker QD, Caster JM, Levin ED, Kuhn CM (2009). Are adolescents more vulnerable to drug addiction than adults? Evidence from animal models. Psychopharmacology (Berl) 206: 1-21.

Spear LP (2000). The adolescent brain and age-related behavioral manifestations. Neurosci Biobehav Rev 24: 417-463.

Vassoler FM, Byrnes EM, Pierce RC (2014). The impact of exposure to addictive drugs on future generations: physiological and behavioral effects. Neuropharmacology 76(Part B): 269-275.

Vathy I (2002). Prenatal opiate exposure: long-term CNS consequences in the stress system of the offspring. Psychoneuroendocrinology 27: 273-283.

Wallace M, Luine V, Arellanos A, Frankfurt M (2006). Ovariectomized rats show decreased recognition memory and spine density in the hippocampus and prefrontal cortex. Brain Res 1126: $176-182$.

Weaver IC, Meaney MJ, Szyf M (2006). Maternal care effects on the hippocampal transcriptome and anxiety-mediated behaviors in the offspring that are reversible in adulthood. Proc Natl Acad Sci USA 103: 3480-3485.

White DA, Michaels CC, Holtzman SG (2008). Periadolescent male but not female rats have higher motor activity in response to morphine than do adult rats. Pharmacol Biochem Behav 89: 188-199.

Zhao C, Li M (2012). Neuroanatomical substrates of the disruptive effect of olanzapine on rat maternal behavior as revealed by c-Fos immunoreactivity. Pharmacol Biochem Behav 103: 174-180.

Supplementary Information accompanies the paper on the Neuropsychopharmacology website (http://www.nature.com/npp) 